\title{
CARLESON MEASURES AND MULTIPLIERS OF DIRICHLET-TYPE SPACES
}

\author{
RON KERMAN AND ERIC SAWYER
}

\begin{abstract}
A function $\rho$ from $[0,1]$ onto itself is a Dirichlet weight if it is

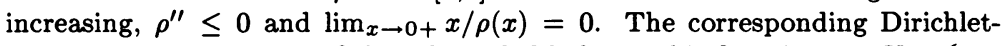
type space, $D_{\rho}$, consists of those bounded holomorphic functions on $U=\{z \in$ C: $|z|<1\}$ such that $\left|f^{\prime}(z)\right|^{2} \rho(1-|z|)$ is integrable with respect to Lebesgue measure on $U$. We characterize in terms of a Carleson-type maximal operator the functions in the set of pointwise multipliers of $D_{\rho}, M\left(D_{\rho}\right)=\{g: U \rightarrow$ C: $\left.g f \in D_{\rho}, \forall f \in D_{\rho}\right\}$.
\end{abstract}

I. Introduction. Let $H(U)$ denote the set of functions holomorphic in the open unit disk $U=\{z \in \mathbf{C}:|z|<1\}$. A function $\rho$ mapping $[0,1]$ onto itself is called a Dirichlet weight if it is increasing, concave in the strong sense that $\rho^{\prime \prime} \leq 0$ and $\lim _{x \rightarrow 0+} x / \rho(x)=0$. Given such a weight, the Dirichlet-type space $D_{\rho}$ consists of those $f \in H(U)$ for which the norm

$$
\|f\|_{\rho}=|f(0)|+\left[\iint_{U}\left|f^{\prime}(z)\right|^{2} \rho(1-|z|) d z\right]^{1 / 2}
$$

is finite; here $d z$ denotes Lebesgue measure on $U$. The main purpose of this paper is to characterize the set $M\left(D_{\rho}\right)$ of pointwise multipliers of $D_{\rho}$, where

$$
M\left(D_{\rho}\right)=\left\{g: U \rightarrow \mathbf{C}: f \in D_{\rho} \text { for all } f \in D_{\rho}\right\} .
$$

Previous results concerning pointwise multipliers of these spaces dealt with the special case

$$
D_{\alpha}=\left\{f \in H(U), f=\sum_{n=0}^{\infty} a_{n} z^{n}:\|f\|_{\alpha}=\left[\sum_{n=0}^{\infty}\left(1+n^{2}\right)^{\alpha}\left|a_{n}\right|^{2}\right]^{1 / 2}<\infty\right\},
$$

$-\infty<\alpha<\infty$; in case $\alpha=1 / 2$ this is the classical Dirichlet space of functions in $H(U)$ whose derivatives are square-integrable on $U$. Taylor [10] described $M\left(D_{\alpha}\right)$ when $\alpha$ lies outside $\left(0,1 / 2\right.$ ]: for $a \leq 0, M\left(D_{\alpha}\right)=H^{\infty}(U)=H(U) \cap L^{\infty}(U)$ while for $\alpha>1 / 2, M\left(D_{\alpha}\right)=D_{\alpha}$ (so that $D_{\alpha}$ is an algebra). D. Stegenga [8] showed that for $\rho_{\alpha}(r)=r^{1-2 \alpha}, \alpha<1$,

$$
\|f\|_{\alpha} \approx|f(0)|+\left[\iint_{U}\left|f^{\prime}(z)\right|^{2} \rho_{\alpha}(1-|z|) d z\right]^{1 / 2}
$$

Received by the editors December 5, 1986.

1980 Mathematics Subject Classification (1985 Revision). Primary 31C25; Secondary 42B25, 42B15.

Research of the first author was supported in part by NSERC grant A4021.

Research of the second author was supported in part by NSERC grant A5149. 
that is, the two terms in (1.2) are equivalent in the sense that each is no larger than a constant multiple of the other, the constants being independent of $f$. (We observe $[0,1 / 2]$ is precisely the range of $\alpha$ for which $\rho_{\alpha}$ is concave.) Stegenga proved $g \in M\left(D_{\alpha}\right), \alpha \in(0,1 / 2]$, if and only if $g \in H^{\infty}(U)$ and

$$
\int_{\bigcup_{j} S\left(I_{j}\right)}\left|g^{\prime}(z)\right|^{2} \rho_{\alpha}(1-|z|) d z \leq C \operatorname{Cap}_{\alpha}\left(\bigcup_{j} I_{j}\right)
$$

for all finite collections of pairwise disjoint subarcs $\left\{I_{j}\right\}$ on the unit circle $T=\{z \in$ C: $|z|=1\}$. Here $S(I)$ denotes the square $\left\{z=r e^{i \theta}: e^{i \theta} \in I\right.$ and $(1-|I|)_{+}<r<$ $1\},|I|$ is the arclength of the subarc $I$, and $\operatorname{Cap}_{\alpha}(E)$ denotes the Bessel capacity of order $\alpha$ of the set $E$.

Our characterization of $M\left(D_{\rho}\right)$ differs from (1.3) in that we replace the capacity by a Carleson-type maximal operator and so are able to test a certain inequality, (1.4), over subarcs of $T$ rather than finite unions of subarcs. This maximal operator, $M_{\rho}$, associates to nonnegative $h$ on $U$ a function $M_{\rho} h$ on $T$ by

$$
\left(M_{\rho} h\right)\left(e^{i \theta}\right)=\sup [|I| \rho(|I|)]^{-1 / 2} \iint_{S(I)} h(z) d z,
$$

the supremum being over all subarcs $I$ of $T$ containing $e^{i \theta}$. Adopting the usual notation, $\chi_{E}$, for the characteristic function of the set $E$, we can now state

THEOREM A. Suppose $\rho$ is a Dirichlet weight. Then $g \in M\left(D_{\rho}\right)$ if and only if $g \in H^{\infty}(U)$ and there exists $C>0$ such that

$$
\begin{array}{r}
\int_{I}\left[M_{\rho}\left(\chi_{S(I)}\left|g^{\prime}(\cdot)\right|^{2} \rho(1-|\cdot|)\right)\left(e^{i \theta}\right)\right]^{2} d \theta \\
\quad \leq C \iint_{S(I)}\left|g^{\prime}(z)\right|^{2} \rho(1-|z|) d z<\infty
\end{array}
$$

for all subarcs $I$ of $T$.

We now outline in some detail the proof of Theorem A. There are two main steps. First, in $\S 2$, Plancherel's theorem is used to show the norm defined at $f(z)=\sum_{n=0}^{\infty} a_{n} z^{n}$ in $D_{\rho}$ by

$$
\|f\|_{\rho}^{*}=\left[\left|a_{0}\right|^{2}+\sum_{n=1}^{\infty} n \rho\left(\frac{1}{n}\right)\left|a_{n}\right|^{2}\right]^{1 / 2}
$$

is equivalent to the one in (1.1). We next observe that, $\rho$ being concave, $n \rho(1 / n)$ is a nondecreasing function of $n$, so $D_{\rho}$ must be contained in the Hardy space $H^{2}(T)=\left\{f \in L^{2}(T): \hat{f}(n)=0\right.$ for $\left.n<0\right\}$. Thus, defining the function $K_{\rho}$ on $T$ in terms of its Fourier coefficients

$$
\hat{K}_{\rho}(n)=\left\{\begin{array}{l}
2, \quad n=0, \\
{[|n| \rho(1 /|n|)]^{-1 / 2}, \quad n \neq 0,}
\end{array}\right.
$$

we can identify $D_{\rho}$ with the space $S_{\rho}$ of Poisson integrals of $K_{\rho}$-potentials of functions in $H^{2}(T)$,

$$
S_{\rho}=\left\{f(z)=\left(P_{r} * K_{\rho} * h\right)\left(e^{i \theta}\right): z=r e^{i \theta}, h \in H^{2}(T)\right\} ;
$$


here, as usual,

$$
(g * h)\left(e^{i \theta}\right)=\frac{1}{2 \pi} \int_{T} g\left(e^{i(\theta-t)}\right) h\left(e^{i t}\right) d t
$$

and $P_{r}(\theta)=\left(1-r^{2}\right) /\left(1-2 r \cos \theta+r^{2}\right)$. Indeed, if $f \in D_{\rho}$, then $f=P_{r} * K_{\rho} * h$ where

$$
\hat{h}(n)= \begin{cases}a_{0} / 2, & n=0 \\ 0, & n<0, \\ n \rho(1 / n) a_{n}, & n>0 .\end{cases}
$$

Second, by [5, Proposition 1.6/4], $M\left(D_{\rho}\right)$ is embedded in $H^{\infty}(U)$. As pointed out on p. 178 of [5], this fact, together with the product rule of differentiation shows $g \in M\left(D_{\rho}\right)$ if and only if $g \in H^{\infty}(U)$ and

$$
\iint_{U}|f(z)|^{2}\left|g^{\prime}(z)\right|^{2} \rho(1-|z|) d z<\infty
$$

for all $f \in D_{\rho}$. The identification of $D_{\rho}$ with $S_{\rho}$ described above then says $g \in$ $M\left(D_{\rho}\right)$ if and only if $g \in H^{\infty}(U)$ and

$$
\iint_{U}\left|\left(P_{r} * K_{\rho} * h\right)\left(e^{i \theta}\right)\right|^{2}\left|g^{\prime}\left(r e^{i \theta}\right)\right|^{2} \rho(1-r) d\left(r e^{i \theta}\right)<\infty
$$

for all $h \in H^{2}(T)$. Taking complex conjugates, it is seen (1.7) would, in fact, hold for all $h \in L^{2}(T)$. By the closed graph theorem, this is equivalent to the existence of $C>0$ such that

$$
\iint_{U}\left|\left(P_{r} * K_{\rho} * h\right)\left(e^{i \theta}\right)\right|^{2} d \mu\left(r e^{i \theta}\right) \leq C \int_{T}\left|h\left(e^{i \theta}\right)\right|^{2} d \theta
$$

for all $h \in L^{2}(T)$, with $d \mu=\left|g^{\prime}(z)\right|^{2} \rho(1-|z|) d z$. Any $\mu \in B(U)$, the class of positive Borel measures on $U$, which satisfies (1.8) will be called a Carleson measure on $S_{\rho}$. The proof of Theorem A is completed in $\S$ III by a characterization of such Carleson measures. This characterization is also used in §IV to give another approach to the "Féjer-Riesz inequality" of Nagel, Rudin and Shapiro [6].

II. Dirichlet-type spaces. In Theorem 2.2 below we require the following result, whose proof can be found, for example, in [2, p. 183].

LEMMA 2.1. Suppose $\left\{c_{n}\right\}_{n=-\infty}^{\infty}$ is an even, nonnegative sequence on $Z$ which is nonincreasing and convex on $Z_{+}$with $\lim _{n \rightarrow \infty} c_{n}=0$. Then the even function $K\left(e^{i \theta}\right)=\sum_{n=-\infty}^{\infty} c_{n} e^{-i n \theta}$ is nonnegative and integrable on $T$.

THEOREM 2.2. Suppose $\rho$ is a Dirichlet weight. Then the function $K_{\rho}$ given through its Fourier coefficients in (1.6) is even, nonnegative and integrable on $T$. The mapping $T_{\rho}$ defined in terms of $K_{\rho}$ by

$$
\left(T_{\rho} h\right)\left(r e^{i \theta}\right)=\left(P_{r} * K_{\rho} * h\right)\left(e^{i \theta}\right), \quad h \in H^{2}(T),
$$

is 1-1 from $H^{2}(T)$ onto $D_{\rho}$. Further, the norm \|\|$_{\rho}^{*}$ of (1.5) induced on $T_{\rho} h \in D_{\rho}$ by taking the $H^{2}(T)$-norm of $h$ is equivalent to the norm \|\|$_{\rho}$ in (1.1).

PROOF. The assertions concerning $K_{\rho}$ follow from Lemma 2.1 once it is observed $c_{n}=[n \rho(1 / n)]^{-1 / 2}, n \geq 1$, is nonincreasing and convex as a consequence of the concavity of $\rho$. 
We next prove $\|\quad\|_{\rho}$ and \|\|$_{\rho}^{*}$ are equivalent, which implies $D_{\rho} \subset T_{\rho}\left(H^{2}(T)\right)$, as was pointed out in $\S 1$. Now, Plancherel's theorem shows that for $f(z)=$ $\sum_{n=0}^{\infty} a_{n} z^{n}$ in $H(U)$

$$
\begin{aligned}
\iint_{U}\left|f^{\prime}(z)\right|^{2} \rho(1-|z|) d z & =\int_{0}^{1} \rho(1-r) r d r \int_{0}^{2 \pi}\left|\sum_{n=1}^{\infty} n a_{n} r^{n-1} e^{i(n-1) \theta}\right|^{2} d \theta \\
& =2 \pi \sum_{n=1}^{\infty} n^{2}\left|a_{n}\right|^{2}\left[\int_{0}^{1} r^{2 n-1} \rho(1-r) d r\right] .
\end{aligned}
$$

We must prove

$$
\int_{0}^{1} r^{2 n-1} \rho(1-r) d r=\int_{0}^{1} \rho(r)(1-r)^{2 n-1} d r \approx n^{-1} \rho(1 / n) .
$$

Since $\rho$ is increasing,

$$
\begin{aligned}
{\left[\frac{(1-1 / n)^{2 n}}{2}\right] n^{-1} \rho(1 / n) } & =\rho(1 / n) \int_{1 / n}^{1}(1-r)^{2 n-1} d r \\
& \leq \int_{1 / n}^{1} \rho(r)(1-r)^{2 n-1} d r \leq \int_{0}^{1} \rho(r)(1-r)^{2 n-1} d r
\end{aligned}
$$

and

$$
\int_{0}^{1 / n} \rho(r)(1-r)^{2 n-1} d r \leq \rho(1 / n) \int_{0}^{1 / n}(1-r)^{2 n-1} d r \leq n^{-1} \rho(1 / n) .
$$

Moreover, $\rho(r) / r$ nonincreasing implies

$$
\int_{1 / n}^{1} \rho(r)(1-r)^{2 n-1} d r \leq n \rho(1 / n) \int_{1 / n}^{1} r(1-r)^{2 n-1} d r .
$$

But, integration by parts, followed by elementary estimates, yields

$$
\int_{1 / n}^{1} r(1-r)^{2 n-1} d r \leq n^{-2}
$$

This completes the proof of the equivalence of the norms.

To see $T_{\rho}$ is $1-1$ onto $D_{\rho}$ we note that as

$$
\left(T_{\rho} h\right)\left(r e^{i \theta}\right)=\left(P_{r} * K_{\rho} * h\right)\left(e^{i \theta}\right)=\sum_{n=-\infty}^{\infty} a_{n} r^{n} e^{i n \theta}
$$

then

$$
[|n| \rho(1 /|n|)]^{1 / 2} a_{n}=[|n| \rho(1 /|n|)]^{1 / 2} \hat{K}_{\rho}(n) \hat{h}(n)= \begin{cases}\hat{h}(n), & n>0 \\ 0, & n<0\end{cases}
$$

III. Carleson measures. In this section we obtain a characterization of the Carleson measures of $S_{\rho}$ and then use it to complete the proof of Theorem A.

Let $K$ be any even, nonnegative function in $L^{1}(T)$. We wish to determine those $\mu \in B(U)$ for which the operator $T_{K}: h \rightarrow P_{r} * K * h$ is bounded from $L^{2}(T)$ to $L^{2}(\mu)$. Indeed, we consider the more general problem in which the index 2 is 
replaced by any fixed $p, 1<p<\infty$. Such a measure $\mu$ will be called a Carleson measure on

$$
S_{K}^{p}=\left\{P_{r} * K * h: h \in L^{p}(T)\right\} .
$$

It turns out to be easier to deal with the equivalent problem for the dual operator $T_{K}^{\prime}$ defined at $\nu \in B(U)$ by

$$
\left(T_{K}^{\prime} \nu\right)\left(e^{i \theta}\right)=\iint_{U}\left(P_{r} * K\right)\left(e^{i(\theta-\phi)}\right) d \nu\left(r e^{i \phi}\right) .
$$

Our result, Theorem 3.1, is given in terms of the maximal operator $M_{K}$ which sends a positive Borel measure $\nu$ on $U$ to a function $M_{K} \nu$ on $T$, with

$$
\left(M_{K} \nu\right)\left(e^{i \theta}\right)=\sup \left[|I|^{-1} \int_{0}^{|I|} K\left(e^{i \phi}\right) d \phi\right] \int_{S(I)} d \nu,
$$

the supremum being over all subarcs $I$ of $T$ containing $e^{i \theta}$. For $K$ even and nonnegative on $T$, let $\tilde{K}\left(e^{i \theta}\right)=\sup _{|\theta| \leq \phi \leq \pi} K\left(e^{i \phi}\right)$ denote the least even nonincreasing majorant of $K$. For $1<p<\infty, p^{\prime}=p /(p-1)$.

THEOREM 3.1. Fix $p \in(1, \infty)$ and suppose $K$ is an even, nonnegative function in $L^{1}(T)$ such that

$$
\int_{0}^{\theta} \tilde{K}\left(e^{i \phi}\right) d \phi \leq C \int_{0}^{\theta} K\left(e^{i \phi}\right) d \phi, \quad 0<\theta<\pi .
$$

Finally, let $\mu \in B(U)$. Then $T_{K}: L^{p}(T) \rightarrow L^{p}(\mu)$ if and only if $C>0$ exists such that

$$
\int_{I}\left[M_{K}\left(\chi_{S(I)} \mu\right)\left(e^{i \theta}\right)\right]^{p^{\prime}} d \phi \leq C \int_{S(I)} d \mu<\infty
$$

for subarcs $I$ of $T$.

The proof of Theorem 3.1 follows the line of argument used by the authors in [4] (see also [3]) to study the $L^{p}$ trace inequality for convolution operators with radially decreasing kernels. The first step is to relate $T_{K}^{\prime}$ and $M_{K}$ (cf. Bonami-Johnson [1]).

THEOREM 3.2. Let $p, K$ and $\tilde{K}$ be as in Theorem 3.1. Then,

(a) There is $C>0$ such that $\left(M_{K} \nu\right)\left(e^{i \theta}\right) \leq C M\left(T_{K}^{\prime} \nu\right)\left(e^{i \theta}\right), e^{i \theta} \in T$, for all $\nu \in B(U)$. Here $M$ is the classical Hardy-Littlewood maximal operator on $T$,

$$
(M g)\left(e^{i \theta}\right)=\sup _{e^{i \theta} \in I}|I|^{-1} \int_{I}\left|g\left(e^{i \phi}\right)\right| d \phi .
$$

(b) There exists $\gamma>1$ and $C^{\prime}>0$ such that for all $\lambda>0$, all $\beta \in(0,1]$ and all $\nu \in B(U)$,

$$
\begin{aligned}
\mid\left\{e^{i \theta}\right. & \left.\in T:\left(T_{\tilde{K}}^{\prime} \nu\right)\left(e^{i \theta}\right)>\gamma \lambda,\left(M_{K} \nu\right)\left(e^{i \theta}\right) \leq \beta \lambda\right\} \mid \\
& \leq C^{\prime} \beta\left|\left\{e^{i \theta} \in T: M\left(T_{\tilde{K}}^{\prime} \nu\right)\left(e^{i \theta}\right)>\lambda\right\}\right| .
\end{aligned}
$$


Proof. (a) Fix a subarc $I$ of $T$. Then,

$$
\begin{aligned}
\int_{I}\left(T_{K}^{\prime} \nu\right)\left(e^{i \theta}\right) d \theta & =\int_{I} d \theta \iint_{U}\left(P_{r} * K\right)\left(e^{i(\theta-\phi)}\right) d \nu\left(r e^{i \phi}\right) \\
& =\iint_{U} d \nu\left(r e^{i \phi}\right) \int_{I}\left(P_{r} * K\right)\left(e^{i(\theta-\phi)}\right) d \theta \\
& \left.\geq \inf _{r i^{i \phi} \in S(I)} \int_{I}\left(P_{r} * K\right)\left(e^{i(\theta-\phi)}\right) d \theta\right] \int_{S(I)} d \nu\left(r e^{i \phi}\right) .
\end{aligned}
$$

However, for $r e^{i \phi} \in S(I)$,

$$
\begin{aligned}
\int_{I}\left(P_{r} * K\right)\left(e^{i(\theta-\phi)}\right) d \theta & =\int_{I} d \theta \int_{T} P_{r}\left(e^{i(\theta-\phi-t)}\right) K\left(e^{i t}\right) d t \\
& =\int_{T} K\left(e^{i t}\right) d t \int_{I} P_{r}\left(e^{i(\theta-\phi-t)}\right) d \theta \\
& \geq \int_{-|I|}^{|I|} K\left(e^{i t}\right) d t \int_{I} P_{r}\left(e^{i(\theta-\phi-t)}\right) d \theta \\
& \geq C \int_{0}^{|I|} K\left(e^{i t}\right) d t
\end{aligned}
$$

since

$$
\int_{I} P_{r}\left(e^{i(\theta-\phi-t)}\right) d \theta \geq \int_{|I|}^{2|I|} P_{r}\left(e^{i s}\right) d s \geq C,
$$

when $(1-|I|)_{+} \leq r<1$ and $\phi+t \in 3 I$. Combining (3.3) and (3.4) yields

$$
|I|^{-1} \int_{I}\left(T_{K}^{\prime} \nu\right)\left(e^{i \theta}\right) d \theta \geq C\left[|I|^{-1} \int_{0}^{|I|} K\left(e^{i t}\right) d t\right] \int_{S(I)} d \nu\left(r e^{i \theta}\right)
$$

for all $I$. Now fix $e^{i \theta} \in T$ and take the supremum in (3.5) over all $I$ containing $e^{i \theta}$ to complete the proof of (a).

(b) Let $\lambda>0$ be given and set

$$
\Omega_{\lambda}=\left\{e^{i \theta} \in T: M\left(T_{\tilde{K}} \nu\right)\left(e^{i \theta}\right)>\lambda\right\} .
$$

Let $\left\{I_{K}\right\}$ be those component subarcs of $\Omega_{\lambda}$ for which $e^{i \theta_{k}} \in I_{k}$ exists such that $\left(M_{K}\right)\left(e^{i \theta_{k}}\right) \leq \beta \lambda$. Fix such a subarc and denote it by $I$. Let $3 I$ be the subarc with the same centre as $I$ but 3 times the length. We have

$$
\begin{gathered}
(3|I|)^{-1}\left[\int_{0}^{3|I|} K\left(e^{i \theta}\right) d \theta\right]\left[\int_{S(3 I)} d \nu\left(r e^{i \phi}\right)\right] \leq \beta \lambda ; \\
|I|^{-1} \int_{I}\left(T_{\tilde{K}}^{\prime} \nu\right)\left(e^{i \theta}\right) d \theta \leq \lambda,
\end{gathered}
$$

since $\left(M_{K} \nu\right)\left(e^{i \theta}\right) \leq \beta \lambda$ for some $e^{i \theta} \in I$ and since $M\left(T_{\tilde{K}}^{\prime} \nu\right) \leq \lambda$ at each end of $I$. Define $\nu_{1}=\left.\nu\right|_{S(3 I)}$ and $\nu_{2}=\nu-\nu_{1}=\left.\nu\right|_{S(3 I)^{c}}$. It will be sufficient to obtain

$$
\left(T_{\tilde{K}}^{\prime} \nu_{2}\right)\left(e^{i \theta}\right) \leq C_{1} \lambda, \quad e^{i \theta} \in I,
$$


for some $C_{1}>0$ independent of $I$. To see this, suppose (3.8) holds and $\gamma>2 C_{1}$. Then,

Now

$$
\begin{aligned}
\mid\left\{e^{i \theta}\right. & \left.\in I:\left(T_{\tilde{K}^{\nu}}^{\prime} \nu\right)\left(e^{i \theta}\right)>\gamma \lambda\right\} \mid \\
& \leq\left|\left\{e^{i \theta} \in I:\left(T_{\tilde{K}}^{\prime} \nu_{1}\right)\left(e^{i \theta}\right)>C_{1} \lambda\right\}\right| \\
& \leq\left(C_{1} \lambda\right)^{-1} \int_{I}\left(T_{\tilde{K}}^{\prime} \nu_{1}\right)\left(e^{i \theta}\right) d \theta .
\end{aligned}
$$

and

$$
\begin{aligned}
\int_{I}\left(T_{\tilde{K}}^{\prime} \nu_{1}\right)\left(e^{i \theta}\right) d \theta & =\int_{I} d \theta \iint_{S(3 I)}\left(P_{r} * \tilde{K}\right)\left(e^{i(\theta-\phi)}\right) d \nu\left(r e^{i \phi}\right) \\
& =\iint_{S(3 I)} d \nu\left(r e^{i \phi}\right) \int_{I}\left(P_{r} * \tilde{K}\right)\left(e^{i(\theta-\phi)}\right) d \theta
\end{aligned}
$$

$$
\begin{aligned}
& \int_{I}\left(P_{r} * \tilde{K}\right)\left(e^{i(\theta-\phi)}\right) d \theta=\int_{I} d \theta \int_{T} P_{r}\left(e^{i t}\right) \tilde{K}\left(e^{i(\theta-\phi-t)}\right) d t \\
& \quad=\int_{T} P_{r}\left(e^{i t}\right) d t \int_{I} \tilde{K}\left(e^{i(\theta-\phi-t)}\right) d \theta \\
& \quad \leq \int_{T} P_{r}\left(e^{i t}\right) d t \int_{-|I| / 2}^{|I| / 2} \tilde{K}\left(e^{i s}\right) d s \leq 2 \int_{0}^{|I|} \tilde{K}\left(e^{i s}\right) d s
\end{aligned}
$$

since $\tilde{K}\left(e^{i s}\right)$ is even when $s \in[-\pi, \pi]$ and nonincreasing when $s \in[0, \pi]$. Combining (3.9), (3.10) and (3.11) we obtain

$$
\begin{aligned}
\mid\left\{e^{i \theta}\right. & \left.\in I:\left(T_{\tilde{K}}^{\prime} \nu\right)\left(e^{i \theta}\right)>\gamma \lambda\right\} \mid \\
& \leq 2\left(C_{1} \lambda\right)^{-1}\left[\int_{0}^{3|I|} \tilde{K}\left(e^{i s}\right) d s\right]\left[\iint_{S(3 I)} d \nu\left(r e^{i \phi}\right)\right] \\
& \leq C C_{1}^{-1} \beta|I|,
\end{aligned}
$$

where the last inequality follows from (3.1) and (3.6). Summing (3.12) over all the $I_{k}$ gives (b).

It remains to prove (3.8). We claim this follows from the fact that

$$
\left(P_{r} * \tilde{K}\right)\left(e^{i(\theta-\phi)}\right) \leq C|I|^{-1} \int_{|\theta-\phi-t| \leq|I|}\left(P_{r} * \tilde{K}\right)\left(e^{i t}\right) d t
$$

whenever $e^{i \theta} \in I$ and $r e^{i \phi} \notin S(3 I)$. For given (3.13), we have

$$
\begin{aligned}
\left(T_{\tilde{K}}^{\prime} \nu_{2}\right)\left(e^{i \theta}\right) & =\iint_{S(3 I)^{c}}\left(P_{r} * \tilde{K}\right)\left(e^{i(\theta-\phi)}\right) d \nu\left(r e^{i \phi}\right) \\
& \leq C|I|^{-1} \iint_{S(3 I)^{c}} d \nu\left(r e^{i \phi}\right) \int_{|\theta-\phi-t| \leq|I|}\left(P_{r} * \tilde{K}\right)\left(e^{i t}\right) d t \\
& \leq C|I|^{-1} \iint_{U} d \nu\left(r e^{i \phi}\right) \int_{|s| \leq|I|}\left(P_{r} * \tilde{K}\right)\left(e^{i(\theta-\phi-s)}\right) d s \\
& =C|I|^{-1} \int_{|s| \leq|I|}\left(T_{\tilde{K}}^{\prime} \nu\right)\left(e^{i(\theta-s)}\right) d s \\
& \leq C \lambda \quad \text { by }(3.7),
\end{aligned}
$$

as required by (3.8). 
To see (3.13) observe that if $r e^{i \phi} \notin S(3 I)$, then either $e^{i \phi} \notin 3 I$, in which case $|I| \leq|\theta-\phi|$ since $e^{i \theta} \in I$; or $0 \leq r<1-3|I|$. In the former case (3.13) holds, since $\left(P_{r} * \tilde{K}\right)\left(e^{i s}\right)$ is even for $s \in[-\pi, \pi]$ and nonincreasing for $s \in(0, \pi]$. In the latter case we use the inequality

$$
P_{r}\left(e^{i t}\right) \leq C|I|^{-1} \int_{|s| \leq|I|} P_{r}\left(e^{i(t-s)}\right) d s, \quad-\pi \leq t \leq \pi, 0<r<1-3|I| .
$$

(For the case $|t| \leq|I|$, use the estimate $P_{r}\left(e^{i u}\right) \approx 1 /(1-r),|u| \leq 2|I|$; for the case $|t|>|I|$, use the fact that $u \rightarrow P_{r}\left(e^{i u}\right)$ is decreasing away from 0 .) Thus the left side of (3.13),

$$
\int_{T} P_{r}\left(e^{i(\theta-\phi-u)}\right) \tilde{K}\left(e^{i u}\right) d u
$$

is dominated by

$$
\begin{aligned}
& C \int_{T}|I|^{-1} \int_{|s| \leq|I|} P_{r}\left(e^{i(\theta-\phi-u-s)}\right) d s \tilde{K}\left(e^{i u}\right) d u \\
& \quad=C|I|^{-1} \int_{|\theta-\phi-t| \leq|I|} d t \int_{T} P_{r}\left(e^{i(t-u)}\right) \tilde{K}\left(e^{i u}\right) d u
\end{aligned}
$$

which equals the right side of (3.13).

The second step in the proof of Theorem 3.1 involves the following analogue of a two-weight norm inequality for maximal operators in [7]; the proof is a straightforward adaptation of ones given in [7] and so is omitted.

THEOREM 3.3. Let $K$ be as in Theorem 3.1 and let $q \in(1, \infty)$. Suppose $\mu \in B(U), \nu \in B(T)$. Then, the inequality

$$
\int_{T}\left[M_{K}(f \mu)\left(e^{i \theta}\right)\right]^{q} d \nu\left(e^{i \theta}\right) \leq C \iint_{U} f\left(r e^{i \phi}\right)^{q} d \nu\left(r e^{i \phi}\right)
$$

holds for all $f \geq 0$ on $U$ if and only if

$$
\int_{I}\left[M_{K}\left(\chi_{S(I)} \mu\right)\left(e^{i \theta}\right)\right]^{q} d \nu\left(e^{i \theta}\right) \leq C \iint_{S(I)} d \mu\left(r e^{i \phi}\right)
$$

for all subarcs $I$ of $T$.

We are now ready to give the

PROOF OF THEOREM 3.1. By duality, $T_{K}: L^{P}(T) \rightarrow L^{P}(\mu)$ if and only if

$$
\int_{T}\left[T_{K}^{\prime}(f \mu)\left(e^{i \theta}\right)\right]^{p^{\prime}} d \theta \leq C \iint_{U} f\left(r e^{i \phi}\right)^{p^{\prime}} d \mu\left(r e^{i \phi}\right)
$$

for all $f \geq 0$ in $L^{p^{\prime}}(\mu)$. Part (a) of Theorem 3.2, together with the Hardy-Littlewood maximal theorem (see $[\mathbf{9}$, p. 5]), shows that

$$
\begin{aligned}
\int_{T}\left[M_{K}(f \mu)\left(e^{i \theta}\right)\right]^{p^{\prime}} d \theta & \leq C \int_{T}\left[M\left(T_{K}^{\prime}(f \mu)\right)\left(e^{i \theta}\right)\right]^{p^{\prime}} d \theta \\
& \leq C \int_{T}\left[T_{K}^{\prime}(f \mu)\left(e^{i \theta}\right)\right]^{p^{\prime}} d \theta
\end{aligned}
$$


Again, by part (b) of Theorem 3.2 and the maximal theorem in [9],

$$
\begin{aligned}
\int_{T}\left[T_{\tilde{K}}^{\prime}(f \mu)\left(e^{i \theta}\right)\right]^{p^{\prime}} d \theta \\
=p^{\prime} \gamma^{p^{\prime}} \int_{0}^{\infty} \lambda^{p^{\prime}-1}\left|\left\{\theta: T_{\tilde{K}}^{\prime}(f \mu)\left(e^{i \theta}\right)>\gamma \lambda\right\}\right| d \lambda \\
\leq C \int_{0}^{\infty} \lambda^{p^{\prime}-1}\left|\left\{M_{K}(f \mu)\left(e^{i \theta}\right)>\beta \lambda\right\}\right| d \lambda \\
\quad+C \beta \int_{0}^{\infty} \lambda^{p^{\prime}-1}\left|\left\{M\left(T_{\tilde{K}}^{\prime} f \mu\right)\left(e^{i \theta}\right)>\lambda\right\}\right| d \lambda \\
\leq C \beta^{-p^{\prime}} \int_{T}\left[M_{K}(f \mu)\left(e^{i \theta}\right)\right]^{p^{\prime}} d \theta+C \beta \int_{T}\left[M\left(T_{\tilde{K}}^{\prime}(f \mu)\right)\left(e^{i \theta}\right)\right]^{p^{\prime}} d \theta \\
\leq C \beta^{-p^{\prime}} \int_{T}\left[M_{K}(f \mu)\left(e^{i \theta}\right)\right]^{p^{\prime}} d \theta+C \beta \int_{T}\left[\left(T_{\tilde{K}}^{\prime}(f \mu)\right)\left(e^{i \theta}\right)\right]^{p^{\prime}} d \theta .
\end{aligned}
$$

Choosing $\beta$ so small that $C \beta<1 / 2$ and subtracting $C \beta \int_{T}\left[T_{\tilde{K}}^{\prime}(f \mu)\left(e^{i \theta}\right)\right]^{p^{\prime}} d \theta$ from both sides of $(3.16)$ yields

$$
\begin{gathered}
\int_{T}\left[T_{K}^{\prime}(f \mu)\left(e^{i \theta}\right)\right]^{p^{\prime}} d \theta \leq \int_{T}\left[T_{\tilde{K}}^{\prime}(f \mu)\left(e^{i \theta}\right)\right]^{p^{\prime}} d \theta \\
\quad \leq C \int_{T}\left[M_{K}(f \mu)\left(e^{i \theta}\right)\right]^{p^{\prime}} d \theta .
\end{gathered}
$$

From (3.15) and (3.17) we conclude that (3.14) holds if and only if

$$
\int_{T}\left[M_{K}(f \mu)\left(e^{i \theta}\right)\right]^{p^{\prime}} d \theta \leq C \iint_{U} f\left(r e^{i \phi}\right)^{p^{\prime}} d \mu\left(r e^{i \phi}\right)
$$

for all $f \geq 0$. Finally, Theorem 3.3 with $d \nu\left(e^{i \theta}\right)=d \theta$ yields the conclusion of Theorem 3.1.

As pointed out in $\S 1, g \in M\left(D_{\rho}\right)$ if and only if $g \in H^{\infty}(U)$ and $T_{K_{\rho}}: L^{2}(T) \rightarrow$ $L^{2}(\mu)$, where $d \mu(z)=\left|g^{\prime}(z)\right|^{2} \rho(1-|z|) d z$. We now know the boundedness of $T_{K_{\rho}}$ is equivalent to (3.2) holding for $K=K_{\rho}$ and $p=2$, provided it can be shown that $K_{\rho}$ satisfies (3.1). We complete the proof of Theorem A by showing (3.1) for $K=K_{\rho}$, as well as the equivalence of $M_{K_{\rho}}$ and $M_{\rho}$ in

LeMma 3.4. Suppose $\rho$ is a Dirichlet weight and let $K=K_{\rho}$ be defined by (1.6). If $\tilde{K}$ denotes the least nonincreasing even majorant of $K$, then one has the equivalences

$$
x^{-1} \int_{0}^{x} K(y) d y \approx x^{-1} \int_{0}^{x_{x}} \tilde{K}(y) d y \approx[x \rho(x)]^{-1 / 2}, \quad x \in[0, \pi] .
$$

ProOF. By the definition of $\tilde{K}, x^{-1} \int_{0}^{x} K(y) d y \leq x^{-1} \int_{0}^{x} \tilde{K}(y) d y$. Summing by parts we obtain $K(x)=\sum_{n=0}^{\infty}(n+1) \Delta^{2} \hat{K}(n) \phi_{n}(x)$, where

$$
\phi_{n}(x)=(n+1)^{-1}\left[\frac{\sin [(n+1) x / 2]}{\sin (x / 2)}\right]^{2}
$$

is the Féjer kernel of order $n$. Thus, recalling that $\{\hat{K}(n)\}$ is convex, we obtain

$$
K(y) \leq \sum_{n=0}^{\infty}(n+1) \Delta^{2} \hat{K}(n) \sup _{|y| \leq z \leq \pi} \phi_{n}(z) .
$$


But, it is easily seen that

$$
\int_{0}^{x}\left[\sup _{|y| \leq z \leq \pi} \phi_{n}(z)\right] d y \leq C \int_{0}^{x} \phi_{n}(y) d y
$$

for $C>0$ independent of $n$. Hence, the averages of $K$ and $\tilde{K}$ are equivalent.

With $N=[1 / x]$,

$$
x^{-1} \int_{0}^{x} K(y) d y \approx \int_{-\pi}^{\pi} \phi_{N}(y) K(y) d y \approx \sum_{|n| \leq[1 / x]}\left(1-\frac{|n|}{N+1}\right) \hat{K}(n)
$$

the last equivalence is a consequence of the general form of Parseval's formula. To complete the proof of the lemma it will be sufficient to prove $x^{-1} \int_{0}^{x} K(y) d y \approx$ $[x \rho(x)]^{-1 / 2}$ for $x$ near 0 , say $x \in(0,1 / 4)$. For such $x$, we have from (1.6) and (3.18)

$$
\begin{aligned}
C^{-1} \sum_{n=1}^{[1 / x] / 2}[n \rho(1 / n)]^{-1 / 2} & \leq x^{-1} \int_{0}^{x} K(y) d y \\
& \leq C \sum_{n=1}^{[1 / x]}[n \rho(1 / n)]^{-1 / 2},
\end{aligned}
$$

or, equivalently (since $\rho$ concave implies $\rho(z) / z$ nonincreasing)

$$
\begin{aligned}
C^{-1} \int_{1}^{1 / 2 x}[y \rho(1 / y)]^{-1 / 2} d y & \leq x^{-1} \int_{0}^{x} K(y) d y \\
& \leq C \int_{1}^{1 / x}[y \rho(1 / y)]^{-1 / 2} d y
\end{aligned}
$$

But, since $\rho(z) / z$ nonincreasing,

$$
\begin{aligned}
\int_{1}^{1 / 2 x}[y \rho(1 / y)]^{-1 / 2} d y & \geq[\rho(2 x) / 2 x]^{-1 / 2}\left(\frac{1}{2 x}-1\right) \\
& \geq 4^{-1}[x \rho(x)]^{-1 / 2}
\end{aligned}
$$

while

$$
\int_{1}^{1 / x}[y \rho(1 / y)]^{-1 / 2} d y \leq \rho(x)^{-1 / 2} \int_{1}^{1 / x} y^{-1 / 2} d y \leq 2[x \rho(x)]^{-1 / 2}
$$

IV. The Féjer-Riesz inequality. Finally, we specialize Theorem 3.3 to the case in which $K=K_{\rho}$, so that $M_{K_{\rho}}$ is equivalent to $M_{\rho} ; \nu$ is Lebesgue measure on $T ; \mu$ is carried by the line segment $L=\{z \in U: \operatorname{Im} z=0,0 \leq \operatorname{Re} z<1\}$.

COROllary 4.5. Suppose $\rho$ is a Dirichlet weight and let $\mu \in B(U)$ be carried by L. Then, $\mu$ is a Carleson measure on $S_{\rho}$ if and only if

$$
\int_{0}^{t}\left[\sup _{x \leq s \leq t}[s \rho(s)]^{-1 / 2} \int_{1-s}^{1} d \mu\right]^{2} d x \leq C \int_{1-t}^{1} d \mu<\infty
$$

whenever $0<t<1$.

Condition (4.1) suggests a natural way to construct a Carleson measure for $S_{\rho}$ which is absolutely continuous with respect to Lebesgue measure on $L$. The idea 
is to suppose equality holds in (4.1) and that the supremum in square brackets on the left side is attained at $s=x$. With $F(t)=\int_{1-t}^{1} d \mu$, this means

$$
\int_{0}^{t}[x \rho(x)]^{-1} F(x)^{2} d x=C F(t), \quad 0<t<1 .
$$

If we further normalize $\mu$ so that $F(1)=1$ and set $C=1$, then $F$ satisfies the boundary value problem

$$
\left\{\begin{array}{l}
F^{\prime}(t)=[t \rho(t)]^{-1} F(t)^{2} \\
F(0+)=0, \quad F(1)=1
\end{array}\right.
$$

whose solution is

$$
F(t)=\left[1+\int_{t}^{1}[s \rho(s)]^{-1} d s\right]^{-1} .
$$

Let

$$
d \mu(t)=d F(t)=[t \rho(t)]^{-1}\left[1+\int_{t}^{1}[s \rho(s)]^{-1} d s\right]^{-2} d t
$$

on $L$. Then (4.1) will hold provided the supremum in square brackets on the left side is attained when $s=x$; that is, provided the function

$$
G(y)=[y \rho(y)]^{-1 / 2} F(y)=[y \rho(y)]^{-1 / 2}\left[1+\int_{y}^{1}[s \rho(s)]^{-1} d s\right]^{-1}
$$

is nonincreasing. While this is not the case, it is true that $G$ is almost decreasing; that is,

$$
G(y) \leq 2 G(x), \quad 0<x \leq y<1,
$$

and this is enough to force (4.1). Since $G(1)=1$ and, by L'Hôpital's rule,

$$
\begin{aligned}
\lim _{y \rightarrow 0+} G(y) & =\lim _{y \rightarrow 0+}\left[\rho(y)+y \rho^{\prime}(y)\right] / 2[y \rho(y)]^{1 / 2} \\
& \geq \lim _{y \rightarrow 0+} 2^{-1}[\rho(y) / y]^{1 / 2}=\infty,
\end{aligned}
$$

it suffices to prove (4.3) when both $x$ and $y$ are critical points of $G$ or $x$ is critical and $y=1$. However, if $G^{\prime}(z)=0$, then

$$
1+\int_{z}^{1}[t \rho(t)]^{-1} d t=2 /\left[\rho(z)+z \rho^{\prime}(z)\right]
$$

So, for $x$ and $y$ critical,

$$
\begin{aligned}
G(y) & =2^{-1}\left[\rho(y)+y \rho^{\prime}(y)\right] /[y \rho(y)]^{1 / 2} \\
& \leq[\rho(y) / y]^{1 / 2} \quad\left(y \rho^{\prime}(y) \leq \rho(y)\right) \\
& \leq[\rho(x) / x]^{1 / 2} \quad(x \leq y) \\
& \leq\left[\rho(x)+x \rho^{\prime}(x)\right] /[x \rho(x)]^{1 / 2} \quad\left(x \rho^{\prime}(x) \geq 0\right) \\
& =2 G(x),
\end{aligned}
$$

and for $x$ critical and $y=1$,

$$
G(y)=1 \leq[p(x) / x]^{1 / 2} \leq 2 G(x) .
$$


To summarize, we have proved

COROllary 4.6 (CF. NAGEL, RUdin AND ShaPIRo [6]). Suppose $\rho$ is a Dirichlet weight and let $\mu$ be the measure carried by $L$ given by (4.2). Then $\mu$ is a Carleson measure for $S_{\rho}$.

\section{REFERENCES}

1. A. Bonami and R. Johnson, Tent spaces based on the Lorentz spaces, Math. Nachr. 132 (1987), 81-99.

2. Y. Katznelson, An introduction to harmonic analysis, Wiley, New York, 1968.

3. R. Kerman and E. Sawyer, Weighted norm inequalities for potentials with applications to Schrodinger operators, Fourier transforms and Carleson measures, Bull. Amer. Math. Soc. (N.S.) 12 (1985), 112-116.

4. (Grenoble) 36 (1986), 207-228.

5. V. G. Maz'ya and T. O. Shaposhnikova, Theory of multipliers in spaces of differentiable functions, Pitman, 1985.

6. A. Nagel, W. Rudin, and J. H. Shapiro, Tangential boundary benaviour of function in Dirichlettype spaces, Ann. of Math. (2) 116 (1982), 331-360.

7. E. Sawyer, A characterization of a two-weight norm inequality for maximal operators, Studia Math. 75 (1982), 1-11.

8. D. A. Stegenga, Multipliers of the Dirichlet space, Illinois J. Math. 24 (1980), 113-139.

9. E. M. Stein, Singular integrals and differentiability properties of functions, Princeton Univ. Press, Princeton, N.J., 1970.

10. G. D. Taylor, Multipliers on $D_{\alpha}$, Trans. Amer. Math. Soc. 123 (1966), 229-240.

Department of Mathematics, Brock University, St. Catharines, Ontario, CANADA

Department of Mathematics and Statistics, MCMaster University, HamilTON, ONTARIO, CANADA 